\title{
Aggression among Chronic Schizophrenic Patients
}

V.M.Samy, M.M.El Hamady, S.T.El Bakry and A.E.Sayer-Dayer

Neuropsychiatry Dept., Faculty of Medicine, Benha Univ., Benha, Egypt

E-Mail:amira_sayerdayer@gmail.com

\begin{abstract}
Patients suffering from schizophrenia are often portrayed in the media as being unpredictably aggressive and violent. Not only the prevalence of violence is similar in psychiatric patients and in the general population, but also many studies proved that aggression levels may be higher in the general population. Many factors, including insufficient social support to mental illnesses, drug abuse, and symptom exacerbations, can precipitate aggressive behavior. Another important major risk factor for aggression is the failure to treat schizophrenic patients adequately.Aggressive behavior and impulsivity are often found in paranoid schizophrenia and can occur during both acute and chronic phases of the illness. Impulsivity is defined as action without planning or reflection, and it seems to be related to a failure of behavioral filtering outside of consciousness. The assessment not only how aggressive patient is, but also how that aggression is manifested by studying aggression-related symptoms, including hostility, anger, and impulsivity.In this cross sectional observational study, 30 patients [16 male\&14 female] aged between $21 \& 56$ years old diagnosed with Schizophrenia and 60 controls were recruited. This sample was totally selected from outpatient clinic of Benha Mental Hospital. All patients in the study were subjected to a detailed history taking, complete general examination, neuropsychiatric clinical assessment, a semistructured interview empathizing the sociodemographic data, the criteria of schizophrenia diagnosis and the Buss and Perry Aggression Questionnaire. The collected data were tabulated and analyzed using SPSS version21 software. Regarding Information obtained from ICD-10 in the studied group and types of schizophrenia: $62.5 \%$ of males and $57 \%$ of females were considered aggressive, while of the control group; $69 \%$ of females and $65 \%$ of males were aggressive. The control sample was almost as aggressive as the sample of patients suffering from chronic schizophrenia. Females and males are similarly aggressive. Patients using combined treatment of atypical antipsychotics and mood stabilizers had higher aggressive scores than who used atypical antipsychotics only. There is not much relationship between marital status and education and aggression. The duration of illness did not affect the type or level of aggression, nor did previous forensic history.
\end{abstract}

Keywords: Anger, Hostility, Aggression, Schizophrenia, victimization, Stigma.

\section{Introduction}

Aggression is any behavior directed toward another individual that is carried out with the immediate intent to cause harm [Andreason][1]. This may be communicated verbally or may manifest in a range of behaviors causing physical or psychological harm towards the self or others, or damage to the environment [2].

Aggression is a common entity in psychiatric disorders, particularly psychotic disorders, often being the first or main symptom for which the patient is admitted or receives medical attention. Various studies report the prevalence of aggression in psychosis patients in the range of $34-70 \%$ [3].

Studies [and meta-analyses] including both twin and adoption samples show that about half [50\%] of the variance in aggressive behavior is explained by genetic influences in both males and females, with the remaining $50 \%$ of the variance being explained by non-shared environmental factors [4].

One of the contextual risk factors for aggression identified by [5] is substance abuse, and the presence of substance abuse increases the risk of violence and aggression, as well as other studies that have found that episodes of violence involving patients with psychiatric disorders [schizophrenia, mania, major depression and bipolar disorder] was five times higher than in the general population.
Schizophrenia is a chronic, complex disorder, in addition to ongoing episodes of psychosis, individuals with schizophrenia frequently experience comorbid conditions such as depression and substance use. Social functioning and life stress also have significant impact on the patient with schizophrenia [6].

Schizophrenic patients have less insight, experience greater thought disorder, and have poorer control of their aggressive impulses. Comorbidity with alcohol or other substances of abuse is frequent and complicates the agitation and the impulsivity. Among patients with schizophrenia, major depression disorder, and bipolar disorder, the risk for homicide was found to be increased with comorbid alcohol abuse or dependence [7].

Patients suffering from schizophrenia are often portrayed in the media as being unpredictably aggressive and impulsive. The result is increased stigmatization and poorer treatment outcomes. Multiple factors, including insufficient social support, substance abuse, and symptom exacerbations, can precipitate aggressive behavior. Moreover, failure to treat schizophrenic patients adequately is a major risk factor for aggression ${ }^{[8]}$.

In terms of the biological understanding, Soyka[9]points out that no single neurobiological theory gained enough support to explain aggressive behavior in schizophrenia. Additionally, neuro-imaging studies reviewed were mixed but overall concluded that deficits in 
the orbito-frontal and temporal cortex may be significant in the neurological underpinnings of aggression.

Schizophrenic patients are more liable to aggression than other populations and there is a strong correlation between the duration of hospital stay and aggression among schizophrenia patients explained [8].

People with, and those who are developing, schizophrenia are at increased risk to engage in aggressive behavior. Some incidents lead to criminal prosecution. Factors contributing to schizophrenia that is preceded by conduct disorder include failing to learn not-to-behave aggressively in early childhood, impairments in understanding emotions in the faces of others, maltreatment, and subsequent re-victimization [10].

\section{Patients and methods}

This is cross sectional descriptive comparative study, where the sample was totally selected from the outpatient clinic of Benha Mental Hospital. The study started on 1st of December 2017 till April 2018. Study subjects were informed of the possibility of using the data obtained for academic purpose. Confidentiality was assured to all participants and data used for this study were stripped of personally identifiable information.

\subsection{Patients}

Study participants were in the age group of 21-56 years. Schizophrenic patients $[\mathrm{n}=30]$ and control subjects $[\mathrm{n}=60]$.Thirty patients [ 16 male\& 14 female] aged between 26 and 55 years old diagnosed as having schizophrenia symptoms joint the study. All patients were selected by considering strict inclusion and exclusion criteria. Patients were selected from the outpatient clinic of Benha Mental Hospital. Sixty staff members from the hospital were also selected randomly but cautiously to match the patients and inclusion and exclusion criteria as well.

\subsection{Methods}

All patients in the study were subjected to a detailed history taking, complete general examination, neuropsychiatric clinical assessment, a semistructured interview emphathizing the sociodemographic data. All patients complaining of any schizophrenic symptoms and meeting the inclusion - exclusion criteria were evaluated clinically and full data was explained and after taking their consent to participate in the study.ICD-10 criteria for diagnosing schizophrenia were performed. This was followed by a complete personal history that took about 56 minutes followed by a clinical examination to confirm the diagnosis. The Buss and Perry Questionnaire [Translated Arabic Version] was used to detect whether there was any type of aggression present.

\subsection{Statistical analysis}

The collected data were tabulated and analyzed using SPSS version 21 software. Categorical data were presented as number and percentages while quantitative data were expressed as mean \pm standard deviation and range. In the statistical comparison between the different groups, the significance of difference was tested using ANOVA test $[\mathrm{F}$ value] \& Kruskall-Wallis test to compare mean of more than two groups of quantitative data of parametric and non-parametric respectively or Fisher exact test [FET] was used to examine the relationship between two qualitative variable when the expected count is less than 5 in more than $20 \%$ of cells. Parametric and non-parametric tests of significance were used appropriately. As $\mathrm{P}$ value $<0.05$ was considered statistically significant.

\section{Results}

In this study, $53.3 \% \%$ of the patients were males and $46,7 \% \%$ were females while $46.7 \%$ of controls were males while $53.3 \%$ were females. All patients were diagnosed as chronic schizophrenic patients, paranoid and residual types [33.3\%] each and simple and undifferentiated types were [16.7\%] each.

The mean age of schizophrenic patients was 41 years with standard deviation 8.6 while of healthy individuals was 34 with standard deviation 8 , with age ranging from 26-55 years and 21-56 years respectively.

The highest percentages of participants in both groups were married. Divorce rates were higher in patients by almost $30 \%$. And more patients were single than were controls.

It is demonstrated that 10 out of 16 [62.5\%] male patients were considered physically aggressive while 9 out of 14 female patients [64.3\%] were.

Regarding the category verbal aggression, $57.1 \%$ of females were verbally aggressive while only $37.5 \%$ of males were.

Regarding Anger and hostility in patients, males were slightly more than females, $62.5 \%, 57.1 \%$ and $68.8 \%$, $64.3 \%$ respectively.

In controls, more females than males were physically aggressive representing $68.8 \%$ versus only $50 \%$ in males. The same was noticed in verbal aggression, as $81.2 \%$ of females versus only $71.4 \%$ of males used verbal methods to represent aggression.

In total, more participants from the control group whether females or males, were considered aggressive in this study sample.

Regarding marital status, the highest percentage of the schizophrenics were married $66.7 \%$ divorced were $6.7 \%$, singles were $23.3 \%$ and widowed were $3.3 \%$ and in controls $65.0 \%$ were married and divorced were $8.3 \%$, and singles were $25.0 \%$.

Regarding subtypes of schizophrenia, There was a significant difference in the prevalence of aggressive traits amongst various schizophrenia subtypes, for example, paranoid patients scored more on all types of aggression [physical, verbal, anger and hostility], with $\mathrm{p}$ value 0.006 for anger, and 0.010 for hostility. This goes with the study 
by Torrey [8] who found that aggressive behavior and impulsivity are often found in paranoid schizophrenia and can occur during both acute and chronic phases of the illness.

Age has negative correlation with anger in control group with $\mathrm{p}$ value 0.009 . This means that the older the person gets, the less angry he become.

Regarding medication in the patients group, it was demonstrated that patients on combined treatment [atypical antipsychotics and mood stabilizers] scored higher on physical aggression, anger, and significantly higher on hostility with $\mathrm{P}$ value 0.027 , than patients on atypical antipsychotics only.

Regarding aggression scores, there was no statistical difference between patients who had previous forensic history and those who didn't.

Comparison between the patient group and the control group as regards the level of aggression, there is no statistical significant difference between the scores of aggression in both groups as regard the level of education, occupation, and marital status.

\section{Types of Schizophrenia}
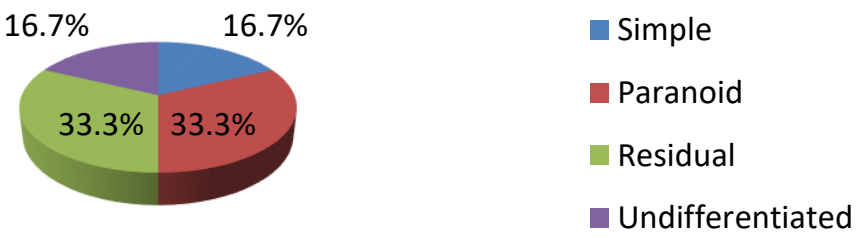

Fig (1) Types of schizophrenia in patients' sample

Table (1) Mental hospital admission and duration of disorder in the patient group.

\begin{tabular}{lccc}
\hline Patient Group & & \multicolumn{2}{l}{} \\
\cline { 3 - 4 } Type of schizophrenia & Paranoid & 10 & $33.3 \%$ \\
& Residual & 10 & $33.3 \%$ \\
& Simple & 5 & $16.70 \%$ \\
& Undifferentiated & 5 & $16.70 \%$ \\
Medications & AAP & 23 & $76.70 \%$ \\
Previous Hospital & AAP + MS & 7 & $23.30 \%$ \\
Admission & Yes & 30 & $100 \%$ \\
& No & 0 & $0 \%$ \\
Duration of illness & $<2$ years & 2 & $6.60 \%$ \\
& $2-5$ years & 4 & $13.30 \%$ \\
Range and mean of illness & $5-10$ years & 6 & $20 \%$ \\
& $>10$ years & 18 & $60 \%$ \\
\hline & Mean \pm SD & & Range of illness in Ys \\
& $13.80 \pm 6.67$ & & $2-25$ \\
\hline
\end{tabular}

Table (2) Relation between types of aggression as regards type of schizophrenia in patient group.

\begin{tabular}{|c|c|c|c|c|c|c|c|c|c|c|}
\hline & \multicolumn{2}{|c|}{$\begin{array}{c}\text { Paranoid } \\
{[\text { No.=10] }}\end{array}$} & \multicolumn{2}{|c|}{$\begin{array}{l}\text { Residual } \\
{[\text { No.=10] }}\end{array}$} & \multicolumn{2}{|c|}{$\begin{array}{c}\text { Simple } \\
{[\text { No.=5] }}\end{array}$} & \multicolumn{2}{|c|}{$\begin{array}{c}\text { Undifferentiated } \\
{[\text { No. }=5]}\end{array}$} & \multicolumn{2}{|c|}{ One way ANOVA } \\
\hline & Mean & SD & Mean & SD & Mean & SD & Mean & SD & $\mathbf{F}$ & P value \\
\hline Physical aggression & 30.50 & 10.96 & 18.30 & 6.57 & 21.60 & 10.95 & 24.00 & 10.49 & 2.815 & 0.059 \\
\hline Verbal aggression & 15.40 & 6.52 & 10.30 & 4.99 & 13.00 & 6.04 & 12.00 & 5.10 & 1.349 & 0.280 \\
\hline Anger & 24.40 & 6.08 & 12.80 & 4.92 & 15.60 & 7.23 & 20.40 & 10.53 & 5.282 & $0.006 * *$ \\
\hline Hostility & 27.90 & 6.49 & 16.60 & 5.48 & 22.80 & 3.27 & 18.80 & 12.15 & 4.678 & $0.010^{*}$ \\
\hline TOTAL & 98.20 & 22.40 & 58.00 & 17.10 & 73.00 & 23.92 & 75.20 & 30.47 & 5.403 & $0.005^{* *}$ \\
\hline
\end{tabular}


Table (3) Comparison between types of aggression in both groups.

\begin{tabular}{lccccc}
\hline & & \multicolumn{2}{c}{$\begin{array}{c}\text { Patient group } \\
\text { No.=30 }\end{array}$} & \multicolumn{2}{c}{$\begin{array}{c}\text { Control group } \\
\text { No.=60 }\end{array}$} \\
\cline { 3 - 6 } & & No. & \% & No. & \% \\
\hline \multirow{2}{*}{ Physical aggression } & Male & 10 & $62.5 \%$ & 14 & $50.0 \%$ \\
& Female & 9 & $64.3 \%$ & 22 & $68.8 \%$ \\
\hline \multirow{2}{*}{ Verbal aggression } & Male & 6 & $37.5 \%$ & 20 & $71.4 \%$ \\
& Female & 8 & $57.1 \%$ & 26 & $81.2 \%$ \\
\hline \multirow{2}{*}{ Anger } & Male & 10 & $62.5 \%$ & 15 & $53.6 \%$ \\
& Female & 8 & $57.1 \%$ & 21 & $65.6 \%$ \\
\hline \multirow{2}{*}{ Hostility } & Male & 11 & $68.8 \%$ & 13 & $46.4 \%$ \\
& Female & 9 & $64.3 \%$ & 15 & $45.5 \%$ \\
\hline \multirow{2}{*}{ Total } & Male & 10 & $62.5 \%$ & 19 & $65.5 \%$ \\
& Female & 8 & $57.1 \%$ & 22 & $68.8 \%$ \\
\hline
\end{tabular}

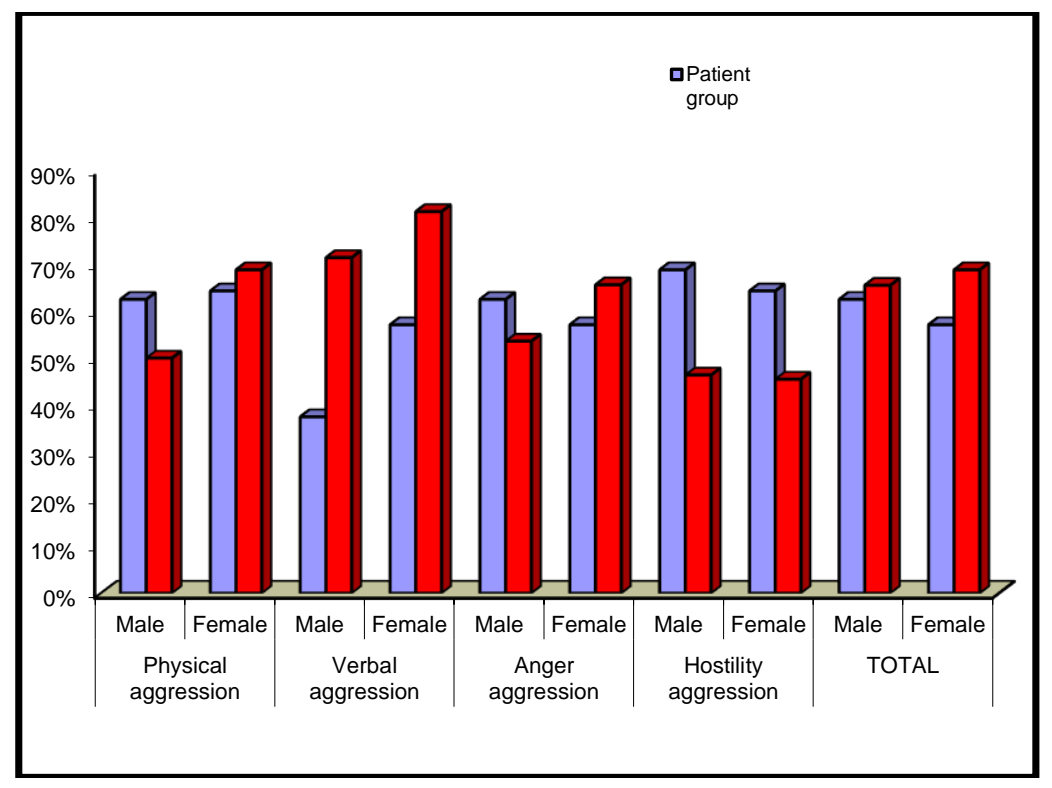

Fig (2) Different aggression types in both genders in the study groups.

\section{Discussion}

This study was done to study Aggression in patients with Chronic Schizophrenia in comparison to healthy individuals.

A total of 90 subjects; 30 patients with Chronic Schizophrenia and 60 staff members were examined.

This short term study was carried out on outpatients in Benha mental hospital, 30 schizophrenic patients and 60 controls were recruited; $57.3 \%$ were males and $42.7 \%$ were females with a mean age of $\pm \mathrm{SD}$ [34.0 \pm 8.06$]$, ages ranged from 21 to 56 years.

In the current study there was no significant difference between different genders which positively agreed with Xiang et al., [2010] who studied the association between genders in schizophrenia using the WHO quality of life questionnaire and found no significant difference between different genders [11].
As regard sex, the patients' sample consisted of 16 males [53.3\%] and 14 females [46.7\%], while the control group consisted of 28 males and 32 females. According to the BPAQ, $62.5 \%$ of the male patients were aggressive $[\mathrm{n}=10]$ and $57.1 \%$ were females $[\mathrm{n}=8]$ while of the control group, $65.5 \%$ [ $n=19]$ were males and $68.8 \%[n=22]$ were females.

The current results showed that the highest percent of patients were ranging from 31-40 years [33\%] and patients ranging from $41-50$ years [33\%] to a lesser extent were patients ranging from 18-30 years [16\%], patients ranging from 51-60 years represented [16\%]. Regarding gender, [53.3\%] were males and [46.7\%] were females. This comes in agreement with Stubbs et al., [12] who demonstrated that the mean age for his study about schizophrenia was 40 years [SD $=11.7$; age range 17-60.3 years], and were predominantly males [64.0\%].In this part 
of the world, more male patients receive mental health attention and care than do female patients. Men and women aren't treated alike, when a woman gets mentally ill, she is either spoiled, psychic, or has hormonal changes. And for a family to admit that she is mentally ill, that means that she will live hidden and in disgrace.

Analyzing the marital status in this study, the patient group showed [20.0\%] single, [53.3\%] married, [26.7\%] divorced and none were widowed. More than half of the patients were still married because in rural areas, there is that misconception of "self-medicating" idea that marriage would make a psychotic patient's life easier. On the other hand, for females in rural villages around Benha city, it is common that women tolerate such disorders and it is a shame to ask for divorce. Most of the divorced and single participants were females. Again, it's a matter of shame and dishonor to be married to a mentally ill woman, especially from the rural areas [like which the study was conducted]. The unawareness and blindness of people makes it difficult for them to get/ remain married. On the other hand, most of the married schizophrenic patients were men; this clearly shows how women are forced [culturally] to take care of her ill husband and never to ask for a divorce because it is culturally unaccepted. Haroun El Rasheed [13] and her colleagues studied 66 schizophrenics admitted into the Institute of Psychiatry, Ain Shams University, diagnosed according to ICD-10. They found that $28[42.42 \%]$ were single, 38 [57.58\%] were married, and 30 [45.45\%] had children.

In the patient group, $62.5 \%$ of males and $57 \%$ of females were considered aggressive. All four subtypes were very close in numbers, except for verbal aggression; it was significantly higher in females [57\%], while only $37 \%$ of males were verbally aggressive."In terms of sex, the results indicated that being female was most associated with verbal aggression against self. This could be interpreted to mean self-deprecating verbalizations, showing anger toward self, making angry noises, and verbal threats of violence towards self" [Al-Sagarat et al.,][14].

In the control group, $69 \%$ of females were aggressive, while only $65 \%$ of males were considered aggressive according to the total aggressive score. According to this study, males were clearly more physically aggressive than females with $P$ value 0.002 and this matches the study by Weiss et al. [15]

Female controls were more verbally aggressive than males, $81 \%$ and $71 \%$ respectively. In addition, more females, almost $66 \%$, but only $53 \%$ of males had positive anger traits. This may be due to the heavy responsibilities working women carry on their shoulders. Some are the only source of income for their families. They use words, screaming, shouting more often than males. They may also be angrier due to raising children which takes most of their time and housework in addition to their morning jobs. Out of the control females, many work as physicians and nurses, who have another item to cause their aggression which is night shifts.

Although many of the participants have economic difficulties; unemployment, bad housings conditions, low salary rates, large family size and extended families, where one or two people may be the source of income for the whole family, there was no significant difference between marital status [single, married, divorced, widowed] in both study groups in relation to aggression. This does not match the results of the Jordanian study Al-Sagarat et al. [14] which revealed that married consumers had a significantly higher frequency of aggression prior to admission than individuals who were single, separated, divorced, or widowed. No significant difference was found for all other types of aggression.

\section{Conclusion and recommendation}

Total aggression percentage in control group was higher than that of patients' group.

Females represent aggression more verbally than males do.

Patients suffering from chronic schizophrenia are wrongly accused of being more aggressive than normal population, and the truth is according to this study, that healthy individuals are.

Based on the findings of the current study the following was recommended:

1- Schizophrenic patients with symptoms of aggression and violence should receive appropriate attention and medications.

2- Schizophrenic patients with paranoid type should have special attention, as they are more risk for development of various types of aggression especially physical aggression and anger.

3- Awareness campaigns against the fake stigma of aggression in schizophrenia.

4- Proper computerized documentation and case records is needed to ease information and data collection for future research. Also a data base between the three main facilities serving mentally ill patients is required.

5- Provide support and guidance, less working hours and creating a positive work environment for mental health workers will reduce stress and eventually aggression rates.

6- It is of extreme importance, to educate all staff of mental health hospitals about the right methods of managing aggression. Burnout and exhaustion of staff should be put into consideration.

7- Future studies are needed to provide more precise estimates of these associations.

\section{Acknowledgement}

We acknowledge all the patients and controls who gave their consent to be part of this study. 
We are thankful to the entire staff of neuropsychiatry department in Benha University Hospital for their consistent support and help.

\section{References}

[1] N.C.Andreasen, D.W.Black, Introductory textbook of psychiatry. Washington,D.C.: American Psychiatric Press,Vol.12(1),PP.54-59.1991.

[2] The National Institute for Health and Care Excellence [NICE] Overview: Violence and Aggression: ShortTerm Management in Mental Health, Health and Community Settings: Guidance." NICE, https: // www. nice. org. uk/ guidance/ ng10, 2015.

[3] Spidel, Alicia, "Aggression and Psychosis: What Is the Role of Abuse History and Symptom Severity in Predicting Compliance?" PsycEXTRA Dataset,Vol. 10,PP.62811,2010.

[4] Tuvblad, Catherine, and A. Baker,"Human Aggression Across the Lifespan." Advances in Genetics Aggression, Vol. ,PP. 171-214. 2011

[5] T.R.Anderson, C.C.Bell, T.E.Powell, L.J.Williamson and Jr. M.A.Blount ,Assessing psychiatric patients for violence. Community Mental Health Journal,Vol.40(4),PP. 379-399,2002.

[6] A. Breier and B.M.astrakhan, characterization of schizophrenic patients who commit suicide. American J psychiatry,Vol. 141,PP.206- 209,2002.

[7] H.Schanda, G.Knecht, D.Schreinzer, Homicide and major mental disorders: a 25-year study. ActaPsychiatr Scand. ,Vol.110,PP.98-107,2004.

[8] E.F.Torrey, Stigma and violence, isn't it time to connect the dots? Schizophr Bull,Vol.37,PP.892$896,2011$.
[9] M.Soyka ,Neurobiology of aggression and violence. Schizophr Bull,Vol.37,PP.913-920, 2011

[10] Hodgins, Sheilagh, “Aggressive Behavior Among Persons With Schizophrenia and Those Who Are Developing Schizophrenia: Attempting to Understand the Limited Evidence on Causality." Schizophrenia Bulletin, Vol. 43(5), PP. 1021-1026,2017.

[11] Y.T.Xiang, Y.Z Weng, C.M.Leung, W.K.Tang, S.S.M.Chan, C.Y.Wang, B.Han, \& G. Ungvari, Gender differences in sociodemographic and clinical characteristic and the quality of life of Chinese schizophrenia patients. Australian and New Zealand Journal of Psychiatry,Vol.44, PP.450-455,2010.

[12] B.Stubbs, J.Firth, A.Berry, F. B.Schuch, S. Rosenbaum, F. Gaughran, D. Vancampfort, How much physical activity do people with schizophrenia engage in? A systematic review, comparative meta-analysis and meta-regression. Schizophrenia Research,Vol.176(2-3),PP.431-440,2016.

[13] A.Haroun El Rasheed, A. Soliman, M. Ghanem, \& S. Effat, Factors affecting drug compliance in an Egyptian sample of relapsing schizophrenic and bipolar affective patients. Current Psychiatry,Vol.8(2),pp 143,2001 .

[14] Al-Sagarat, Y. Ahmad, "Prevalence of Aggressive Behaviours among Inpatients with Psychiatric Disorders: A Case Study Analysis from Jordan." Wiley Online Library, John Wiley \& Sons, Vol.10,PP.118-120,2015.

[15] E.M.Weiss, C.G.Kohler, C.M.Brensinger, W.B.Bilker, J. Loughead, M.Delazer \& K.A.Nolan , Gender differences in facial emotion recognition in persons with chronic schizophrenia. European Psychiatry, Vol.22(2), PP.116-122,2017. 